\title{
Habit of Bathing or Showering Is Beneficially Associated With Body Weight and Abdominal Circumference in Patients With Type 2 Diabetes
}

\author{
Hisayuki Katsuyama a, Hidetaka Hamasaki ${ }^{\text {a, b }}$, Hidekatsu Yanai ${ }^{a}$ c
}

\section{To the Editor}

Non-exercise activity thermogenesis (NEAT) is the energy expenditure of all physical activities other than sporting-like exercise, and includes daily physical activities such as working, taking a bath and dancing [1]. We developed the original questionnaire to evaluate NEAT in Japanese people, based on 2011 Compendium of Physical Activities produced by American College of Sports Medicine [2], by considering Japanese life-style, habits and culture [3]. The questionnaire consisted of 11 question items about locomotive activities such as waling and 25 question items about non-locomotive activities such as housekeeping and occupational activities [3]. We evaluated each questionnaire item with a score of $1-3$ points in order of levels of daily physical activity and then added up the scores to determine the NEAT score. The validity of our NEAT score was confirmed by the triaxial accelerometer [4].

We previously reported that higher NEAT score was beneficially associated with insulin resistance, abdominal circumference, serum lipids, glucose metabolism, blood pressure, and the degree of diabetic nephropathy and diabetic neuropathy, in patients with type 2 diabetes [3,5,6].

Frequent bathing is a characteristic habit of Japanese people. Therefore, our NEAT questionnaires include the item about taking a bath or shower [3]. The question item No. 33 of NEAT score asks you "How often do you take a bath (or shower)?" If you answer "A lot (once or more a day)", you can get 3 points. If you answer "Sometimes (once or more a week)", you can get 2 points. If you answer "Hardly ever", you can get only one point.

To understand a significance of habit of bathing or showering on metabolic parameters, we retrospectively studied a

Manuscript submitted June 12, 2018, accepted June 18, 2018

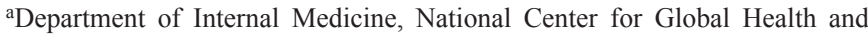
Medicine Kohnodai Hospital, Chiba, Japan

bHamasaki Clinic, Kagoshima, Japan

${ }^{\mathrm{c} C}$ Corresponding Author: Hidekatsu Yanai, Department of Internal Medicine, National Center for Global Health and Medicine Kohnodai Hospital, 1-7-1 Kohnodai, Ichikawa, Chiba 272-8516, Japan.

Email: dyanai@hospk.ncgm.go.jp

doi: https://doi.org/10.14740/jocmr3510w difference in metabolic parameters between patients who obtained 3 points and patients who obtained $1-2$ points in the item of taking a bath (shower) of NEAT score in patients with type 2 diabetes. Furthermore, we studied the correlation between points in the item of taking a bath (shower) of NEAT score and metabolic parameters. Comparison between two groups was analyzed by the Student's $t$-test and chi-squared test, and correlation was analyzed by the Spearman's rank correlation. This study was approved by the Institutional Ethics Committee in National Center for Global Health and Medicine, and was also performed in accordance with the Declaration of Helsinki.

We found 87 eligible patients, whose clinical characteristics and metabolic parameters were shown in Table 1. A difference in metabolic parameters between patients who obtained 3 points and patients who obtained $1-2$ points in the question item of taking a bath (shower) of NEAT score among all subjects was shown in Table 2. Body weight and abdominal circumference were significantly lower in patients who obtained 3 points than in patients who obtained $1-2$ points. Total NEAT score was significantly higher in patients who obtained 3 points than in patients who obtained $1-2$ points. Prevalence of mental disorders was significantly lower in patients who obtained 3 points than in patients who obtained $1-2$ points. The points in the item of taking a bath (shower) of NEAT score were significantly and positively correlated with total NEAT score and negatively correlated with abdominal circumference in all subjects (Table 3).

Body weight, body mass index and abdominal circumference were significantly lower in patients who obtained 3 points than in patients who obtained $1-2$ points in female subjects (Table 4). Total NEAT score was significantly higher in patients who obtained 3 points than in patients who obtained 1 - 2 points. The points in the item of taking a bath (shower) of NEAT score were significantly and positively correlated with total NEAT score and negatively correlated with abdominal circumference in female subjects (Table 5). There were no significant differences in any metabolic parameters between patients who obtained 3 points and patients who obtained $1-2$ points in male subjects.

Abdominal circumference was significantly lower in patients who obtained 3 points than in patients who obtained $1-2$ points in female subjects who had not taken oral anti-diabetic drugs and insulin therapy (Table 6). Total NEAT score was significantly higher in patients who obtained 3 points than in 
Table 1. Clinical Characteristics and Metabolic Parameters of Subjects $(n=87)$

\begin{tabular}{ll}
\hline Sex (male/female) & $44 / 43$ \\
Age (years) & $62.3 \pm 14.0$ \\
Body height $(\mathrm{cm})$ & $160 \pm 9$ \\
Body weight $(\mathrm{kg})$ & $71.4 \pm 11.8$ \\
Body mass index $\left(\mathrm{kg} / \mathrm{m}^{2}\right)$ & $24.9 \pm 5.4$ \\
Abdominal circumference (cm) & $90.6 \pm 13.0$ \\
Plasma glucose (mg/dL) & $182 \pm 88$ \\
HbAlc $(\%)$ & $7.5 \pm 1.7$ \\
HDL-C (mg/dL) & $51 \pm 13$ \\
LDL-C (mg/dL) & $122 \pm 31$ \\
TG (mg/dL) & $165 \pm 88$ \\
Oral anti-diabetic drugs (n, \%) & $39(45 \%)$ \\
Insulin therapy (n, \%) & $8(9 \%)$ \\
\hline Mental disorders (n, \%) & $28(32 \%)$ \\
\hline
\end{tabular}

patients who obtained $1-2$ points. The points in the item of taking a bath (shower) of NEAT score were significantly and positively correlated with total NEAT score and negatively correlated with abdominal circumference in female subjects who had not taken oral anti-diabetic drugs and insulin therapy (Table 7).

The present study showed that habit of bathing or showering is beneficially associated with body weight and abdominal circumference in patients with type 2 diabetes, especially, in female patients. Even after the exclusion of influences of antidiabetic treatments, these beneficial effects of habit of bathing or showering on abdominal circumference remained in female subjects. However, these beneficial effects of habit of bathing or showering were not observed in male subjects. The same discrepancy between men and women was observed in pre-
Table 3. Correlation Between Points in the Item of Taking a Bath (Shower) of NEAT Score and Metabolic Parameters Among All Subjects

\begin{tabular}{lll}
\hline & Correlation coefficients & P values \\
\hline Age & 0.148 & 0.171 \\
Total NEAT score & 0.520 & $<0.001$ \\
Body weight & -0.189 & 0.087 \\
Body mass index & -0.176 & 0.112 \\
Abdominal circumference & -0.328 & 0.009 \\
Plasma glucose & 0.030 & 0.783 \\
HbA1c & 0.121 & 0.266 \\
HDL-C & 0.137 & 0.216 \\
LDL-C & 0.006 & 0.959 \\
TG & -0.079 & 0.470 \\
\hline
\end{tabular}

vious studies $[3,5]$. Briefly, the NEAT score was negatively correlated with waist circumference and positively correlated with high-density lipoprotein (HDL)-C levels in only women [3]. The NEAT score was significantly and negatively correlated with urinary albumin creatinine ratio (UACR) and positively correlated with coefficient of variation of R-R intervals (CVRR) in only women [5]. Any favorable effects of NEAT score on waist circumference, HDL-C, UACR and CVRR were not observed in male patients with type 2 diabetes $[3,5]$. We thought that this discrepancy between men and women was due to that the NEAT score included many activities which are mainly performed by women. However, this hypothesis cannot explain present result.

In this study, the points in the item of taking a bath (shower) of NEAT score were significantly and positively correlated with total NEAT score, suggesting that the points in the item of taking a bath may reflect total NEAT score very well. The points in the item of taking a bath of NEAT score

Table 2. A Difference in Metabolic Parameters Between Patients Who Obtained 3 Points and Patients Who Obtained 1 - 2 Points in the Item of Taking a Bath (Shower) of NEAT Score Among All Subjects

\begin{tabular}{llll}
\hline Score of taking a bath (shower) & $\mathbf{3}$ points & $\mathbf{1 , 2}$ points & P values \\
\hline $\mathbf{n}$ & $\mathbf{5 7}$ & $\mathbf{3 0}$ & Male/female, 14/16 \\
\hline Age (years) & Male/female, 30/27 & $62.3 \pm 9.2$ & 0.310 \\
Body weight $(\mathrm{kg})$ & $63.4 \pm 14.9$ & $68.2 \pm 15.1$ & 0.040 \\
Body mass index $\left(\mathrm{kg} / \mathrm{m}^{2}\right)$ & $60.4 \pm 15.6$ & $26.5 \pm 6.0$ & 0.075 \\
Abdominal circumference $(\mathrm{cm})$ & $24.2 \pm 5.0$ & $96.5 \pm 13.5$ & 0.016 \\
Total NEAT score & $87.9 \pm 11.8$ & $50.5 \pm 9.8$ & $<0.001$ \\
Plasma glucose (mg/dL) & $62.3 \pm 9.2$ & $183 \pm 96$ & 0.951 \\
\hline HbA1c (\%) & $182 \pm 84$ & $7.2 \pm 1.5$ & 0.309 \\
HDL-C (mg/dL) & $7.6 \pm 1.7$ & $49 \pm 9$ & 0.186 \\
LDL-C (mg/dL) & $53 \pm 14$ & $120 \pm 32$ & 0.662 \\
TG (mg/dL) & $123 \pm 30$ & $175 \pm 89$ & 0.475 \\
Mental disorders (n, \%) & $161 \pm 87$ & $15(50 \%)$ & 0.009 \\
\hline
\end{tabular}


Table 4. A Difference in Metabolic Parameters Between Patients Who Obtained 3 Points and Patients Who Obtained 1 - 2 Points in the Item of Taking a Bath (Shower) of NEAT Score Among Female Subjects $(n=43)$

\begin{tabular}{llll}
\hline Score of taking a bath (shower) & $\mathbf{3}$ points & $\mathbf{1 , 2}$ points & P values \\
\hline $\mathbf{n}$ & $\mathbf{2 7}$ & $\mathbf{1 6}$ & 0.276 \\
\hline Age (years) & $63.2 \pm 17.7$ & $57.6 \pm 12.1$ & 0.039 \\
Body weight $(\mathrm{kg})$ & $58.6 \pm 14.6$ & $69.6 \pm 17.2$ & 0.050 \\
Body mass index $\left(\mathrm{kg} / \mathrm{m}^{2}\right)$ & $25.0 \pm 5.5$ & $28.9 \pm 6.05$ & $<0.001$ \\
Abdominal circumference (cm) & $90.6 \pm 9.1$ & $104.6 \pm 9.8$ & $<0.001$ \\
Total NEAT score & $78.3 \pm 9.3$ & $64.8 \pm 10.1$ & 0.895 \\
Plasma glucose (mg/dL) & $170 \pm 72$ & $167 \pm 62$ & 0.592 \\
\hline HbAlc $(\%)$ & $7.5 \pm 1.5$ & $7.2 \pm 1.3$ & 0.066 \\
HDL-C (mg/dL) & $57 \pm 16$ & $48 \pm 8$ & 0.503 \\
LDL-C (mg/dL) & $121 \pm 30$ & $128 \pm 35$ & 0.276 \\
TG (mg/dL) & $162 \pm 92$ & $194 \pm 79$ & 0.063 \\
\hline Mental disorders (n, \%) & $9(33 \%)$ & $10(63 \%)$ & \\
\hline
\end{tabular}

Table 5. Correlation Between Points in the Item of Taking a Bath (Shower) of NEAT Score and Metabolic Parameters Among Female Subjects

\begin{tabular}{lll}
\hline & Correlation coefficients & P values \\
\hline Age & 0.214 & 0.168 \\
Total NEAT score & 0.610 & $<0.001$ \\
Body weight & -0.244 & 0.119 \\
Body mass index & -0.232 & 0.139 \\
Abdominal circumference & -0.570 & 0.001 \\
Plasma glucose & 0.087 & 0.585 \\
HbA1c & 0.111 & 0.478 \\
HDL-C & 0.261 & 0.095 \\
LDL-C & -0.148 & 0.345 \\
TG & -0.150 & 0.336 \\
\hline
\end{tabular}

Table 6. A Difference in Metabolic Parameters Between Patients Who Obtained 3 Points and Patients Who Obtained 1 - 2 Points in the Item of Taking a Bath (Shower) of NEAT Score Among Female Subjects Who Had Not Taken Oral Anti-Diabetic Drugs and Insulin Therapy $(\mathrm{n}=22)$

\begin{tabular}{llll}
\hline Score of taking a bath (shower) & $\mathbf{3}$ points & $\mathbf{1 , 2}$ points & P values \\
\hline $\mathbf{n}$ & $\mathbf{1 2}$ & $\mathbf{1 0}$ & 0.807 \\
\hline Age (years) & $61.3 \pm 17.9$ & $58.0 \pm 13.3$ & 0.268 \\
Body weight $(\mathrm{kg})$ & $59.5 \pm 14.9$ & $70.1 \pm 20.4$ & 0.285 \\
Body mass index $\left(\mathrm{kg} / \mathrm{m}^{2}\right)$ & $25.4 \pm 6.0$ & $29.3 \pm 7.7$ & 0.034 \\
Abdominal circumference $(\mathrm{cm})$ & $90.1 \pm 11.0$ & $105.4 \pm 10.8$ & 0.005 \\
Total NEAT score & $63.1 \pm 7.0$ & $51.0 \pm 8.6$ & 0.639 \\
Plasma glucose (mg/dL) & $178 \pm 68$ & $166 \pm 74$ & 0.470 \\
HbAlc $(\%)$ & $7.6 \pm 1.4$ & $7.2 \pm 1.5$ & 0.062 \\
HDL-C (mg/dL) & $61 \pm 16$ & $47 \pm 8$ & 0.455 \\
LDL-C (mg/dL) & $139 \pm 33$ & $128 \pm 32$ & 0.391 \\
TG (mg/dL) & $164 \pm 102$ & $212 \pm 82$ & 0.069 \\
Mental disorders (n, $\%)$ & $8(80 \%)$ & \\
\hline
\end{tabular}


Table 7. Correlation Between Points in the Item of Taking a Bath (Shower) of NEAT Score and Metabolic Parameters Among Female Subjects Who Had Not Taken Oral Anti-Diabetic Drugs and Insulin Therapy

\begin{tabular}{lll}
\hline & Correlation coefficients & P values \\
\hline Age & 0.131 & 0.562 \\
Total NEAT score & 0.637 & 0.010 \\
Body weight & -0.158 & 0.482 \\
Body mass index & -0.127 & 0.574 \\
Abdominal circumference & -0.562 & 0.023 \\
Plasma glucose & 0.167 & 0.458 \\
HbA1c & 0.186 & 0.407 \\
HDL-C & 0.363 & 0.097 \\
LDL-C & 0.185 & 0.409 \\
TG & -0.219 & 0.327 \\
\hline
\end{tabular}

were significantly and negatively correlated with abdominal circumference in female subjects, indicating that taking a bath itself may be beneficially associated with abdominal circumference. Each activity included in the NEAT score may give a greater influence on metabolic parameters in women than in men.

Although the evidence on effects of bathing on metabolic parameters is very limited, we cannot deny the possibility that bathing directly improved body weight and abdominal circumference. The physical activity intensity of bathing is 1.5 metabolic equivalents (METs). A $50 \mathrm{~kg}$-person who takes a bath for 30 min every day consumes more energy as compared with the same weighted person who takes a bath once a week, by approximately $236.25 \mathrm{kcal} /$ week. Shimodozono et al reported that leptin significantly increased after a single warm-water bath, and adiponectin showed a slight, but not significant, increase after a single warm-water bath [7]. Thermal therapy such as sauna was suggested to be a promising therapy for patients with lifestyle-related diseases [8].

This study presents one more interesting fact that patients with mental disorders are unlikely to take a bath. We previously reported that NEAT is significantly lower in type 2 diabetic patients with mental disorders than in those without mental disorders [9]. These results suggest that the intervention to each item in the NEAT score may be useful to manage metabolic disorders in patients with mental disorders.

In conclusion, the grasp of the bathing habit is useful to consider the daily physical activities and the influence of lifestyle habits on metabolic abnormalities.

\section{Conflict of Interest}

The authors declare that they have no conflicts of interest concerning this article.

\section{References}

1. Levine JA, Vander Weg MW, Hill JO, Klesges RC. Nonexercise activity thermogenesis: the crouching tiger hidden dragon of societal weight gain. Arterioscler Thromb Vasc Biol. 2006;26(4):729-736.

2. Ainsworth BE, Haskell WL, Herrmann SD, Meckes N, Bassett DR, Jr., Tudor-Locke C, Greer JL, et al. 2011 Compendium of Physical Activities: a second update of codes and MET values. Med Sci Sports Exerc. 2011;43(8):1575-1581.

3. Hamasaki H, Yanai H, Mishima S, Mineyama T, Yamamoto-Honda R, Kakei M, Ezaki O, et al. Correlations of nonexercise activity thermogenesis to metabolic parameters in Japanese patients with type 2 diabetes. Diabetol Metab Syndr. 2013;5(1):26.

4. Hamasaki H, Yanai H, Kakei M, Noda M, Ezaki O. The validity of the non-exercise activity thermogenesis questionnaire evaluated by objectively measured daily physical activity by the triaxial accelerometer. BMC Sports Sci Med Rehabil. 2014;6:27.

5. Hamasaki H, Yanai H, Kakei M, Ezaki O, Noda M. Nonexercise activity thermogenesis is associated with markers for diabetic microangiopathy in Japanese female patients with type 2 diabetes. Int J Cardiol. 2013;168(5):48364837.

6. Yanai H, Adachi H, Masui Y, Katsuyama H, Kawaguchi A, Hakoshima M, Waragai Y, et al. Exercise therapy for patients with type 2 diabetes: a narrative review. J Clin Med Res. 2018;10(5):365-369.

7. Shimodozono M, Matsumoto S, Ninomiya K, Miyata R, Ogata A, Etoh S, Watanabe S, et al. Acute effects of a single warm-water bath on serum adiponectin and leptin levels in healthy men: a pilot study. Int J Biometeorol. 2012;56(5):933-939.

8. Biro S, Masuda A, Kihara T, Tei C. Clinical implications of thermal therapy in lifestyle-related diseases. Exp Biol Med (Maywood). 2003;228(10):1245-1249.

9. Hamasaki H, Ezaki O, Yanai H. Nonexercise activity thermogenesis is significantly lower in type 2 diabetic patients with mental disorders than in those without mental disorders: a cross-sectional study. Medicine (Baltimore). 2016;95(2):e2517. 